\title{
Convolution surfaces with varying radius: Formulae for skeletons made of arcs of circles and line segments
}

\author{
Alvaro Javier Fuentes Suárez*† and Evelyne Hubert ${ }^{\ddagger}$ \\ Inria Méditerranée, France
}

\section{Introduction}

Shape representation based on skeletons have a major role in geometric modeling and animation in Computer Graphics [10]. Skeletons of 3D shapes are made of curves and surfaces, with a preference for the former. Several techniques are used to create a surface enclosing a volume around a skeleton. In this paper we focus on convolution surfaces [5]. This technique provides surfaces with good mathematical features stemming from their definition. It is for instance used in interactive modeling environments $[2,41]$, sketch based modeling $[1,39]$, and applied to model natural shapes $[16,40]$.

Convolution surfaces are the level sets of a convolution function that results from integrating a kernel function $K$ along the skeleton $\mathcal{S}$. The mathematical smoothness of the surface obtained depends only on the smoothness of the kernel. In practice, a kernel function (power inverse, Cauchy, compact support, ...) is selected so as to have closed form expressions for the convolution functions associated to basic skeleton elements (line segments, triangles, ....). Since the additivity property of integration makes the convolution function independent of the partition of the skeleton, skeletons are partitioned and approximated by a set of basic elements. The convolution function for the whole skeleton is obtained by adding the convolution functions of the constitutive basic elements. See for instance [5, 9, 17, 20, 21, 22, 32, 33, 36, 37].

Line segments are the most commonly used 1D basic skeleton elements. When a skeleton consists of curves with high curvature or torsion, its approximation might require a great number of line segments for the convolution surface to look as intended. In this paper we take the stance that arcs of circles form a very interesting class of basic skeleton elements in the context of convolution. This was already argued in [21] for planar skeleton curves. But this can be made more general. Indeed any space curve can be approximated by circular splines in a $\mathcal{G}^{1}$ fashion [28, 34]. A lower number of basic skeleton elements are then needed to obtain an appealing convolution surface, resulting into better visual quality at lower computational cost.

\footnotetext{
${ }^{\dagger}$ alvaro.fuentes-suarez@inria.fr

${ }_{\ddagger}^{\ddagger}$ evelyne.hubert@inria.fr

*This project has received funding from the European Union's Horizon 2020 research and innovation programme under the Marie Skłodowska-Curie grant agreement No 675789.
}

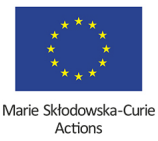


To model a wider variety of shapes it is necessary to vary the thickness around the skeleton. Several approaches have been suggested: weighted skeletons [19, 20, 22], varying radius [17], scale invariant integral surfaces [37], the latter two actually providing a more intrinsic formulation. While general closed form formulae were obtained for weighted line segments in [19], there has been a lack of generality in terms of closed form formulae for convolution with varying radius, or scale, over line segments and, even more so, over arcs of circles. This paper addresses this very issue for the family of power inverse kernels, of even degree. For a family of compact support kernels, the formulae are analogous for the convolution of line segments, but are substantially more complicated in the convolution of arcs of circle. We thus chose not to present them explicitly in this article. Yet these formulae can be obtained with the advanced computer algebra technique that we present in this paper and the power of which we illustrate on power inverse kernels.

As initiated in $[18,19]$, the generality for closed form formulae of the convolution function associated to line segments and arcs of circle for a varying radius or scale is offered in terms of recurrence formulae. Closed form formulae allow efficient evaluation. With recurrence formulae we elegantly reach a higher level of generality: with a simple code we can use kernels of any degree. As detailed and illustrated in [19], the code can be automatically generated and optimized within a computer algebra software like Maple. This contrasts with previous works where the formulae for the convolution functions had to be implemented individually for kernels of every degree. The recurrence formulae in $[18,19]$ mostly drew on integral functions that appear in some classical tables. An innovative approach is taken in this paper. The recurrence formulae we present in this paper are obtained through Creative Telescoping, an active topic of research in computer algebra. A limited set of pointers is $[7,13,25]$ as they provide the background to available software. We introduce the subject and show how this technique can be applied in the context of convolution surfaces.

In Section 2, we provide the definition of convolution surfaces associated to a skeleton made of curves, with varying thickness. In Section 3 we introduce Creative Telescoping and describe how it can be used to obtain recurrence formulae for convolution fields. In Section 4 we examine the convolution of line segments with varying thickness, providing the closed form formulae for the convolution functions. In Section 5 we turn to arcs of circles. In Section 6 we demonstrate the interest of having arcs of circles as basic skeleton elements for convolution and discuss future directions.

Acknowledgements: we want to thank Shaoshi Chen, Bruno Salvy, Alin Bostan, Louis Dumont, Frédéric Chyzak and Christoph Koutschan for several enlightening discussions on Creative Telescoping over the years, as well as making their fantastic software available.

\section{Convolution surfaces}

In this section we recall the basics of convolution surfaces. We first discuss families of kernels that arose in the computer graphics literature. Thereafter, we shall mainly focus on the family of power inverse kernels. We then define the convolution function generated by a bounded regular curve and provide alternative definitions of convolution that allow the modeling of shapes with varying thickness around the skeleton. 


\subsection{Kernels}

The kernels in use in the literature are given by functions $K: \mathbb{R}^{+} \rightarrow \mathbb{R}^{+}$that are at least continuously differentiable. The argument is the distance between a point in space and a point on the skeleton. Those kernels are decreasing functions on $\mathbb{R}^{+}$and strictly decreasing when non zero: $K(r)>0 \Rightarrow K^{\prime}(r)<0$.

The first convolution surfaces [5] were based on the Gaussian kernel $r \mapsto e^{-s r^{2}}$ (also in [4]) that depends on a parameter $s>0$. The difficulty in evaluating the resulting convolutions prompted the introduction of kernels that provided closed form expressions for the convolution functions associated to basic skeleton elements. [32, 33] promoted the Cauchy kernel $r \mapsto \frac{1}{\left(1+\sigma r^{2}\right)^{2}}$ after [35] introduced the inverse function $r \mapsto \frac{1}{r}$. For faster convolution [9, 17] introduced the power inverse cube kernel $r \mapsto \frac{1}{r^{3}}$. [20] also exhibited the benefit of using the quintic inverse $r \mapsto \frac{1}{r^{5}}$. With the power inverse kernels and the Cauchy kernels, the whole skeleton influences the convolution function at a point, even if infinitesimally. In order to limit the zone of influence of each point of the skeleton, compact support kernels were introduced, mostly as piecewise polynomial functions. To some extent, they allow to limit the unwanted bulges or blending (Figure 3). Their use nonetheless necessitates to determine the geometry of the intersection of the skeleton with spheres.

The family of compact support kernels used in [18] is indexed by $i \in \mathbb{N} \backslash\{0\}$ and given by

$$
\boldsymbol{k}_{R}^{i}: r \mapsto \begin{cases}\left(1-\left(\frac{r}{R}\right)^{2}\right)^{\frac{i}{2}} & \text { if } r<R \\ 0 & \text { otherwise }\end{cases}
$$

To obtain convolution surfaces that are at least continuously differentiable only $\boldsymbol{k}_{R}^{i}$ for $i \geq 3$ should be considered. For $i<3, \boldsymbol{k}_{R}^{i}$ is not differentiable at $r=R$. The case $i=4$ is actually the case considered in $[23,33]$. As we increase $i$ though, we obtain smoother shapes.

This paper is mostly concerned with the family of power inverse kernels though. They are indexed by $i \in \mathbb{N} \backslash\{0\}$ and given by

$$
\boldsymbol{p}^{i}: r \mapsto\left(\frac{1}{r}\right)^{i}
$$

The convolution surfaces obtained with a power inverse kernel always enclose the skeleton since these functions tend to infinity when approaching the skeleton. As exploited in $[18,19]$ the closed form formulae of convolution functions using the family of Cauchy kernels $\boldsymbol{c}_{\sigma}^{i}: r \mapsto\left(\frac{1}{1+\sigma r^{2}}\right)^{\frac{i}{2}}$ differ only slightly from the ones using power inverse kernels.

\subsection{Notations}

Typically $P=\left(p_{1}, p_{2}, p_{3}\right)^{T} \in \mathbb{R}^{n}$ represents a point in space and $A=\left(a_{1}, a_{2}, a_{3}\right)^{T}, B=$ $\left(b_{1}, b_{2}, b_{3}\right)^{T}$ represent the end points of a line segment $[A B]$ or arc of circle $\widehat{A^{O B}}$ with center $O$. The straight line through $A$ and $B$ is denoted as $(A B)$. Then $\overrightarrow{A P}$ represent the vector from $A$ to $P$. In the following $\vec{u}=\left(u_{1}, u_{2}, u_{3}\right)^{T}, \vec{v}=\left(v_{1}, v_{2}, v_{3}\right)^{T} \in \mathbb{R}^{n}$ also represent vectors. The scalar product of two vectors $\vec{u}=\left(u_{1}, u_{2}, u_{3}\right)^{T}$ and $\vec{v}=\left(v_{1}, v_{2}, v_{3}\right) \in \mathbb{R}^{n}$ is then $\vec{u} \cdot \vec{v}=u_{1} v_{1}+u_{2} v_{2}+u_{3} v_{3}$. The distance between two points $A$ and $B$ is denoted as $|A B|=\sqrt{\left(b_{1}-a_{1}\right)^{2}+\left(b_{2}-a_{2}\right)^{2}+\left(b_{3}-a_{3}\right)^{2}}$ 

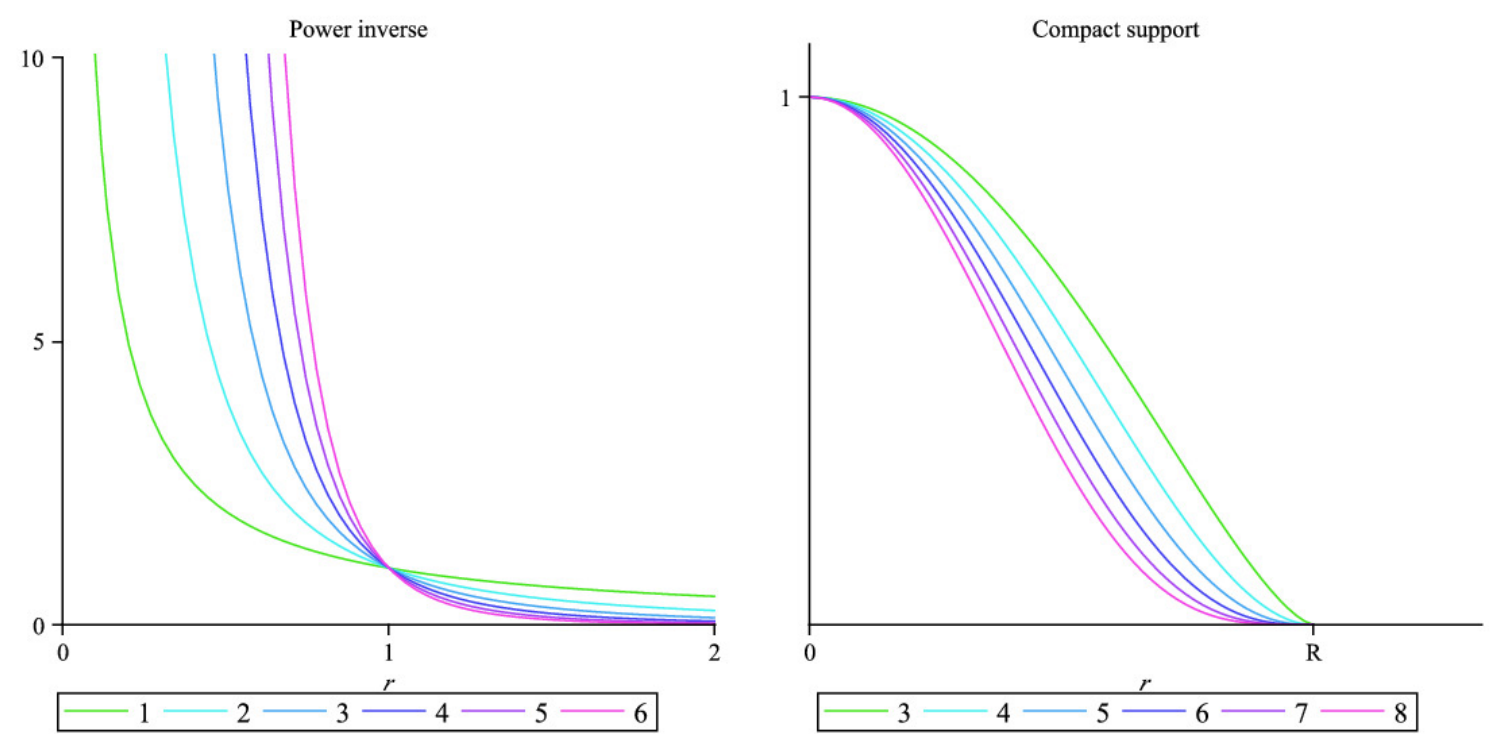

Figure 1: The graphs of the kernel functions $\boldsymbol{p}^{i}$ and $\boldsymbol{k}_{R}^{i}$, varying $i$.

\subsection{Convolution of regular curves}

We assume here that the skeleton can be partitioned into regular patches defined by bounded regular curves parametrized by an interval $[a, b]$ of $\mathbb{R}$. The convolution function for the skeleton is then obtained by summing the convolution functions for each of those patches.

Consider a parametrized curve $\Gamma:[a, b] \subset \mathbb{R} \rightarrow \mathbb{R}^{3}$. It is a regular curve if $\Gamma$ is continuously differentiable and $\Gamma^{\prime}$ does not vanish. The infinitesimal arc-length is then $\left|\Gamma^{\prime}(t)\right| \mathrm{d} t$. The convolution function based on $\mathcal{S}=\Gamma([a, b])$ at a point $P \in \mathbb{R}^{3}$ is then defined by

$$
\mathcal{C}_{\Gamma}^{K}(P)=\int_{a}^{b} K(|P \Gamma(t)|)\left|\Gamma^{\prime}(t)\right| \mathrm{d} t .
$$

The integral is independent of the (regular) parametrization of the curve used. Convolution functions with a power inverse kernel $K=\boldsymbol{p}^{i}$ are infinitely differentiable outside of the curve $\Gamma([a, b])$. Convolution functions with a compact support kernel $K=\boldsymbol{k}_{R}^{i}$ are $\left\lfloor\frac{i-1}{2}\right\rfloor$ continuously differentiable. As the inverse image of a closed set by a $k$-continuously differentiable map, $k \geq 1$, the resulting convolution surfaces $\left\{P \in \mathbb{R}^{3} \mid \mathcal{C}_{\Gamma}^{K}(P)=\kappa\right\}$ are closed (in a topological sense) and smooth, provided $\kappa$ is not a critical value ${ }^{1}$ of $\mathcal{C}_{\Gamma}^{K}$. It is the boundary of a smooth 3 -dimensional manifold $V_{\kappa}=$ $\left\{P \in \mathbb{R}^{3} \mid \mathcal{C}_{\Gamma}^{K}(P) \geq \kappa\right\}$. Furthermore $V_{\kappa}$ and $V_{\kappa^{\prime}}$ are diffeomorphic provided that there is no critical values in the interval $\left[\kappa, \kappa^{\prime}\right]\left[27\right.$, Theorem 3.1]. With the power inverse kernel, the skeleton is in $V_{\kappa}$ for any $\kappa>0$. This is not always the case with a Cauchy or compact support kernel; $V_{\kappa}$ can even be the empty set for too high values of $\kappa$.

\footnotetext{
${ }^{1}$ A critical point of a function $f:(x, y, z) \mapsto f(x, y, z)$ is a point $\left(x_{0}, y_{0}, z_{0}\right)$ at which the gradient $\left(f_{x}, f_{y}, f_{z}\right)$ of $f$ vanishes. A critical value of $f$ is the value $f\left(x_{0}, y_{0}, z_{0}\right)$ of $f$ at a critical point $\left(x_{0}, y_{0}, z_{0}\right)$.
} 


\subsection{Discussion on the choice of a kernel}

With compact support kernels $\boldsymbol{k}_{R}^{i}$ the smoothness of the convolution surface increases with $i$. With power inverse kernels $\boldsymbol{p}^{i}$ the convolution functions are smooth at all points outside the skeleton. Yet, as $i$ increases, the convolution surface is sharper around the skeleton. This is illustrated in Figure 2.
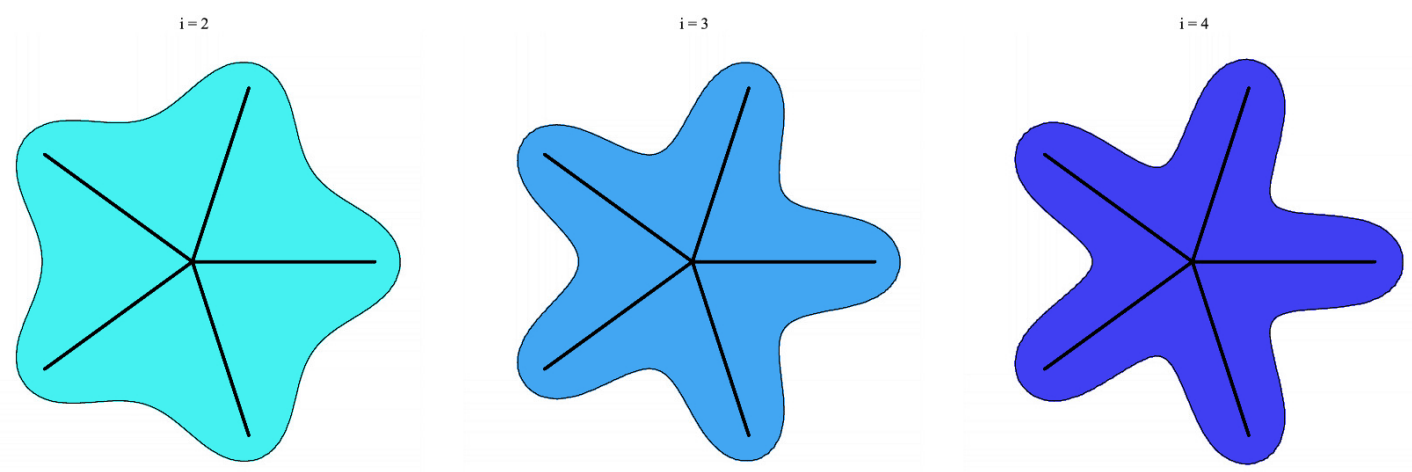

Figure 2: Convolution curves for a set of segments with power inverse kernel $\boldsymbol{p}^{2}, \boldsymbol{p}^{3}, \boldsymbol{p}^{4}$. The convolution function for the whole set is obtained as the sum of the convolution functions for each line segment. The level set is adapted to have identical thickness at the tips. Note that sharpness increases from left to right.

When the convolution function has a critical point, chances are that there is a change in the topology of the convolution surface as it goes through the critical value [27]. This is illustrated in Figure 3 with a skeleton made of two line segments. The convolution function has a critical point and the convolution surface through this point has a singularity. The corresponding level set is a transition from bulging to blending, from two connected components to a single component. Figure 3 also illustrates the fact that compact support kernels allow to dismiss the influence of skeleton elements that are at distance more than $R$, thus avoiding some of the bulging and blending that appear for the kernels with infinite support kernel.

\subsection{Varying thickness}

Several alternatives have been introduced for varying the thickness of the convolution surface along the skeleton. A first idea was to use a weight function along the skeleton [5]. For a skeleton given by a regular curve $\Gamma:[a, b] \rightarrow \mathbb{R}^{3}$, one uses a weight function $w:[a, b] \rightarrow \mathbb{R}$. The convolution function is then defined as

$$
\mathcal{C}_{\Gamma, w}^{K}(P)=\int_{a}^{b} w(t) K(|P \Gamma(t)|)\left|\Gamma^{\prime}(t)\right| \mathrm{d} t .
$$

The convolution function is now dependent on the parametrization used for the skeleton curve.

Polynomial weights were studied in [19, 20, 21, 22], where closed-form formulae was obtained for particular cases of weighted convolution. The drawback of this approach was illustrated in [19, Figure 9]: the influence of the weight diminishes as the degree of the kernel increases. Alternative more intrinsic formulations were proposed in [17, 37]. 

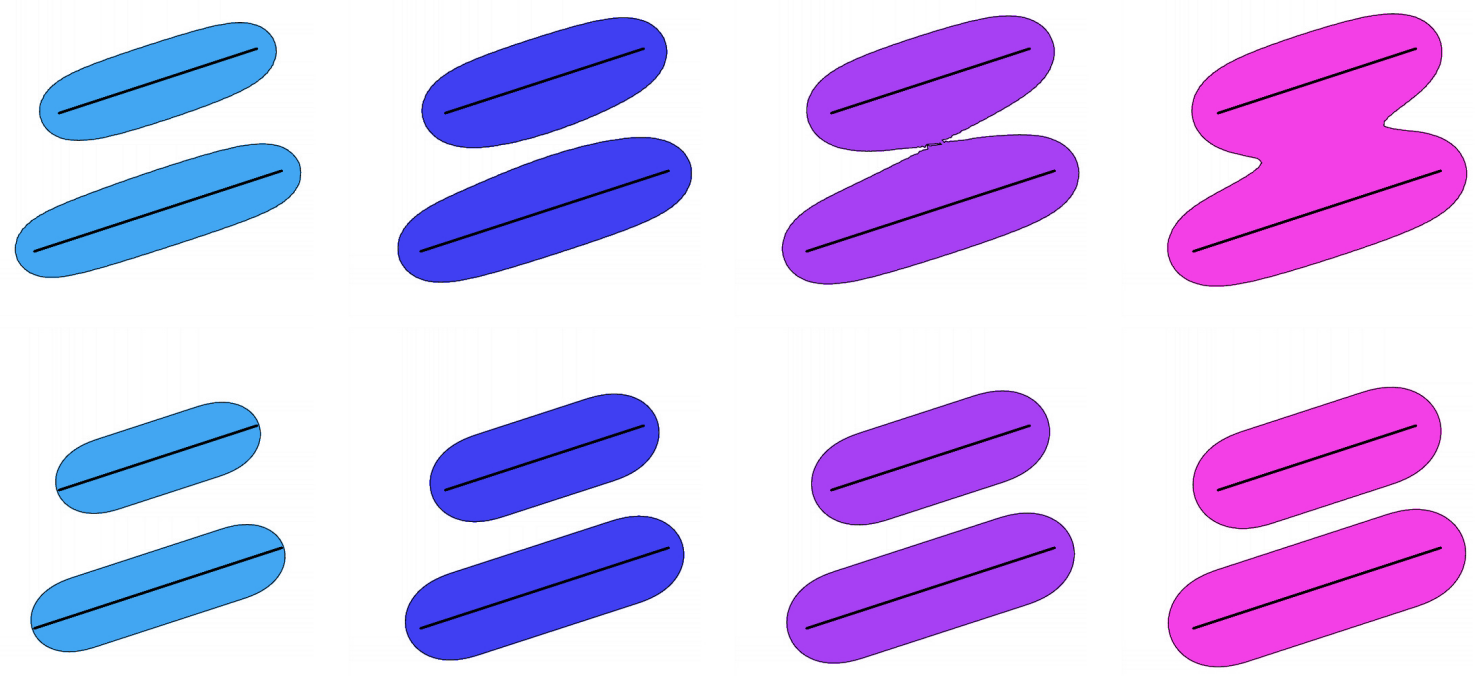

Figure 3: Convolution curves based on two parallel segments. The top line uses the cubic inverse kernel $\boldsymbol{p}^{3}$ and the bottom line a compact support kernel $\boldsymbol{k}_{R}^{3}$. Columns correspond to an identical sought thickness. For the cubic inverse kernel, a bulge and then a blend appear between the line segments, while the convolution surfaces get only thicker with the compact support kernel.

\subsubsection{Varying radius as proposed by Hornus et al. [17]}

In [17] Hornus et al. proposed a different way of computing the convolution function. This allows the user to modify the shape of the final surface according to a radius assigned to each point in the skeleton: the distance is divided by the radius at the corresponding point in the skeleton. For a skeleton curve $\Gamma:[a, b] \rightarrow \mathbb{R}^{3}$ the radius is given by a function $\rho:[a, b] \rightarrow \mathbb{R}^{+}$. The convolution function is then defined by

$$
\mathcal{H}_{\Gamma, \rho}^{K}(P)=\int_{a}^{b} K\left(\frac{|P \Gamma(t)|}{\rho(t)}\right)\left|\Gamma^{\prime}(t)\right| \mathrm{d} t .
$$

If additional precaution are not imposed on the radius function $\rho$, this convolution function depends on the parametrization of the curve $\Gamma$. In practice it makes sense to have a radius function that is approximately linear in the arc-length.

\subsubsection{SCALIS as proposed by Zanni et al. [37]}

An alternative convolution introduced in [37] allows to properly model shapes with pieces at different scales. This lead to the name SCALe invariant Integral Surfaces (SCALIS). For a regular curve $\Gamma:[a, b] \subset \mathbb{R} \rightarrow \mathbb{R}^{3}$, the convolution function is

$$
\mathcal{S}_{\Gamma, \lambda}^{K}(P)=\int_{a}^{b} K\left(\frac{|P \Gamma(t)|}{\lambda(t)}\right) \frac{\left|\Gamma^{\prime}(t)\right|}{\lambda(t)} \mathrm{d} t
$$

where $\lambda:[a, b] \rightarrow \mathbb{R}^{+}$is called the scale function. Notice that $\lambda$ plays a similar role as $\rho$ in the formulation by Hornus. To apprehend all the good features of this new definition of convolution 
surface, the reader is referred to [36,37]. Let us just observe the case where $\lambda$ is a constant. If we write $\lambda \cdot P$ and $\lambda \cdot \Gamma$ for the point and the regular curve obtained through a homothety (a.k.a homogeneous dilatation or scaling) with ratio $\lambda$, then

$$
\mathcal{S}_{\lambda \cdot \Gamma, \lambda}^{K}(\lambda \cdot P)=\mathcal{C}_{\Gamma}^{K}(P) \quad \text { or equivalently } \quad \mathcal{S}_{\Gamma, \lambda}^{K}(P)=\mathcal{C}_{\lambda^{-1} \cdot \Gamma}^{K}\left(\lambda^{-1} \cdot P\right) .
$$

This implies that the convolution surface of equation $\mathcal{S}_{\lambda \cdot \Gamma, \lambda}^{K}(P)=c$ is homothetic to the convolution surface $\mathcal{C}_{\Gamma}^{K}(P)=c$ with a ratio $\lambda$.

\section{Determining general closed form formulae through recurrence}

Closed-form formulae for convolution fields have been studied for several skeleton primitives and kernels $[18,38,19,20,21,22,33,36]$. Given an appropriate parametrization of a skeleton primitive and a kernel, the closed form formulae for the convolution field is very often obtained thanks to symbolic integration [8], which is implemented in computer algebra software as Maple or Mathematica. The field function for the pair consisting of the skeleton primitive and kernel can then be implemented with a view on optimizing its evaluation cost. As different kernels change the properties of the convolution surface, even if lightly, it is interesting to offer, in the same geometric modeling software, alternative kernels for convolution. The same is true for skeleton primitives. As different kernels may bring related closed form formulae one seeks to optimize the code by taking advantage of the common subexpressions [22, Equations 11-14]. A greater level of generality was offered with the new approach in $[18,19]$ where common subexpressions were encapsulated into low order recurrence formulae: kernels were grouped into families, each indexed by an integer, and, for each family, recurrence relationships were exhibited for the convolution function field, indexed by the same integer. The recurrence relationships that appeared in $[18,19]$ mostly came from tables of known integrals, which made this strategy uneasy to generalize.

We present here a technique that allows to obtain algorithmically the recurrence formulae on the convolution fields for families of kernels and a given skeleton primitive. This unified approach relies on Creative Telescoping and we think it is a valuable contribution of ours of realizing how to apply this technique in the context of convolution surfaces. Within computer algebra software, recurrence relationships can be translated into optimized $\mathrm{C}$ code, as illustrated in [19]. But this approach should be balanced against the use of highly accurate numerical integration routines based on quadratures [31]. Indeed, some closed form formulae might be judged too complex to result in efficient code for evaluating the convolution functions.

In this section we give a brief introduction to Creative Telescoping (CT) and illustrate how to use some specialized software for our purpose. In Section 4 and Section 5 we show some specific recurrences we obtained with $\mathrm{CT}$ for line segments and arcs of circle with power inverse and compact support kernels. All the recurrences we provide in this article, as well as the recurrence equations, can be checked by a simple differentiation.

\subsection{Creative Telescoping}

We introduce here Creative Telescoping. This is an active research field [12], with new algorithms and new applications appearing every year. For a complete (but still gentle) introduction we refer the reader to [25], and to [14] for a formal development of the ideas we present here. 
Creative Telescoping comes in several guises depending on the application. We want to find recurrence relations for the integrals in the convolution field. For this case CT works with differential $\left(D_{x}\right)$ and shift $\left(S_{n}\right)$ operators. These operators act by differentiating or incrementing by one (in the respective variable) the input expression $f$, i.e. $D_{x} f(x, n)=\frac{\partial}{\partial x} f(x, n)$ and $S_{n} f(x, n)=f(x, n+1)$. Here $x$ and $n$ stand for continuous and discrete variables respectively.

Higher order linear operators are constructed as elements of the vector space $\mathbb{O}$ spanned by the symbols of the form $D_{x}^{\alpha_{1}} D_{y}^{\alpha_{2}} \ldots S_{n}^{\beta_{1}} S_{m}^{\beta_{2}} \ldots$ with coefficients in $\mathbb{F}=\mathbb{K}(x, y, \ldots, n, m, \ldots)$, the field of rational functions over a field $\mathbb{K}$ (of characteristic 0 ). $\mathbb{O}$ is actually a $\mathbb{F}$-algebra $\mathbb{F}\left\langle D_{x}, D_{y}, \ldots, S_{n}, S_{m}\right\rangle$ in which the generators $\left(D_{x}, D_{y}, \ldots, S_{n}, S_{m}\right)$ commute pairwise (for instance $D_{x} S_{n}=S_{n} D_{x}$ ) but the commutation of an operator with an element in the field is not trivial:

$$
D_{x} p=p D_{x}+\frac{\partial}{\partial x} p \quad \text { and } \quad S_{n} p=\left.p\right|_{n \rightarrow n+1} S_{n} .
$$

$(\mathbb{O}$ is a so called Ore algebra. Ore algebras are defined for operators that generalize both derivations and shifts [29]. In this context one introduces the concept of $\partial$-finite functions [14]. These are often called holonomic functions though there are subtleties between the two notions. Creative Telescoping takes $\partial$-finite functions as input. Basically, a $\partial$-finite function $f$ is uniquely and well defined as the zero of a set of linear operators in $\mathbb{O}$ with prescribed values for a finite subset of its low order derivatives or shifts (the initial conditions). It is then the case that for any operator $\partial$ (of the form $D_{x}$ or $S_{n}$ ) there is an integer $m$ such that $\left\{\partial^{k} f \mid k=0 . . m\right\}$ are linearly dependent over $\mathbb{F}$.

For illustration consider

$$
f(t, i, k)=\frac{(\lambda+\delta t)^{k}}{\left(a t^{2}-2 b t+c\right)^{i}}, \text { with } a, b, c, \delta, \lambda \in \mathbb{R} .
$$

For the operators $D_{t}, S_{i}$ and $S_{k}$ with $\mathbb{F}=\mathbb{R}(t, i, k)$, we see that $f$ is $\partial$-finite by observing that

$$
S_{k} f=\frac{(\lambda+\delta t)^{k+1}}{\left(a t^{2}-2 b t+c\right)^{i}}=(\lambda+\delta t) f, \quad S_{i} f=\frac{(\lambda+\delta t)^{k}}{\left(a t^{2}-2 b t+c\right)^{i+1}}=\frac{1}{a t^{2}-2 b t+c} f
$$

and

$$
D_{t} f=\left(\frac{\delta k}{\lambda+\delta t}-2 i \frac{a t-b}{a t^{2}-2 b t+c}\right) f
$$

Given a complete set of operators annihilating the function, the purpose of Creative Telescoping is to produce operators $P \in \mathbb{O}$ such that

$$
P=\mathcal{L}-D_{t} C \text { and } \quad P f=0
$$

with $\mathcal{L}, C \in \mathbb{O}$ and where $\mathcal{L}$ depends neither on $D_{t}$ nor on $t$. That is $\mathcal{L}$ is a linear combination of $S_{i}^{\beta_{1}} D_{k}^{\beta_{2}}$ over $\mathbb{K}(i, k)$. Because of its total independence on $t, \mathcal{L}$ commutes with an integration operator with respect to $t$ so that

$$
0=\int_{t_{0}}^{t_{1}} P f d t=\int_{t_{0}}^{t_{1}} \mathcal{L} f d t-\int_{t_{0}}^{t_{1}} D_{t} C f d t=\mathcal{L}\left(\int_{t_{0}}^{t_{1}} f d t\right)-[C f]_{t=t_{0}}^{t=t_{1}} .
$$

Thus $F(i, k)=\int_{t_{0}}^{t_{1}} f(t, i, k) d t$ satisfies the recurrence $\mathcal{L} F(i, k)=g\left(t_{1}\right)-g\left(t_{0}\right)$, where $g=C f$. The operator $\mathcal{L}$ is called the telescoper and $C$ the certificate. We thus have obtained a recurrence 
relationship on the functions $F(i, k)=\int_{t_{0}}^{t_{1}} f(t, i, k) d t$. For a given skeleton primitive and a family of kernels, the convolution fields are functions of this type. Creative Telescoping thus provides a general unified approach to obtain the recurrence formulae on these.

Note that Creative Telescoping refers to a panel of algorithms. There is no canonical output. For instance one might trade a low order telescoper for a telescoper with lower degree coefficient [11], or a telescoper computed with a more efficient heuristic [24]. Furthermore, specialized lower complexity algorithms exist for subclasses of functions, like rational, hypergeometric or hyperexponential functions $[6,7]$. The function $f$ we started with is actually hypergeometric since

$$
\frac{D_{t} f}{f} \in \mathbb{F}, \quad \frac{S_{i} f}{f} \in \mathbb{F}, \quad \text { and } \quad \frac{S_{k} f}{f} \in \mathbb{F} .
$$

\subsection{Practical use}

There are several implementations of CT available, a (perhaps limited) set is Mgfun ${ }^{2}$ by F. Chyzak (in Maple), HolonomicFunctions ${ }^{3}$ by C. Koutschan (in Mathematica), and MixedCT ${ }^{4}$ by L. Dumont (in Maple). The description of the algorithms implemented are in the respective publications $[14,24,7]$. Here, as Maple users, we describe an example of use with Mgfun, following up on the running example of previous paragraph.

The operators $D_{t}, S_{i}, S_{k}$ are expressed in Mgfun by the $\mathrm{t}:$ : diff, i : : shift, and k : : shift directives. In order to get recurrence formulae for the integral

$$
F(i, k)=\int_{t_{0}}^{t_{1}} f(t, i, k) d t=\int_{t_{0}}^{t_{1}} \frac{(\lambda+\delta t)^{k}}{\left(a t^{2}-2 b t+c\right)^{i}} d t,
$$

one simply calls the command creative_telescoping $(f(t, i, k),[k:: \operatorname{shift}, i:: \operatorname{shift}], t:: \operatorname{diff})$. It is indeed practical that an appropriate system of linear operators annihilating $f$ is computed internally. The class of expressions for which this works is given by the closure properties of $\partial$-finite functions [14] and accounted for in the documentation of the command dfinite_expr_to_sys in the Mgfun package.

The command creative_telescoping ( $f(t, i, k),[k:: \operatorname{shift}, i::$ shift $], t:: \operatorname{diff})$ outputs two pairs $(\mathcal{L}, C)$. To interpret the output of creative_telescoping the reader must refer to Mgfun documentation. The first pair consists of

$$
\begin{aligned}
\mathcal{L}_{1}= & 2 i\left(a c-b^{2}\right)\left(\lambda^{2} a+2 \lambda b \delta+\delta^{2} c\right) S_{i}-a(2 i-2-k)(a \lambda+\delta b) S_{k} \\
& +\left(2 b^{2}(i-k-1)-a c(2 i-k-1)\right) \delta^{2}-2 a b \lambda(k+1) \delta-a^{2} \lambda^{2}(k+1)
\end{aligned}
$$

and

$$
C_{1}=(\lambda+\delta t)\left(a b t-2 b^{2}+a c\right) \delta+a \lambda(a t-b),
$$

while the second pair consists of

$$
\mathcal{L}_{2}=a(k-2 i+3) S_{k}^{2}+2(i-k-2)(a \lambda+b \delta) S_{k}+(k+1)\left(a \lambda^{2}+2 b \delta \lambda+\delta^{2} c\right)
$$

\footnotetext{
${ }^{2}$ https://specfun.inria.fr/chyzak/mgfun.html

${ }^{3}$ http://www.risc.jku.at/research/combinat/software/ergosum/RISC/HolonomicFunctions.html

${ }^{4}$ http://mixedct.gforge.inria.fr
} 
and

$$
C_{2}=-\delta(\lambda+\delta t)\left(a t^{2}-2 b t+c\right) .
$$

This latter, for instance, translates to the following recurrence, which will be used in Section 4:

$a(k-2 i+3) F(i, k+2)+2(i-k-2)(a \lambda+b \delta) F(i, k+1)+(k+1)\left(a \lambda^{2}+2 b \delta \lambda+\delta^{2} c\right) F(i, k)=\left[\frac{-\delta(\delta t+\lambda)^{k+1}}{\left(a t^{2}-2 b t+c\right)^{i-1}}\right]_{t_{0}}^{t_{1}}$.

Note that this latter telescoper involves $S_{k}$ and not $S_{i}$. The set of pairs $\left\{\left(\mathcal{L}_{1}, C_{1}\right),\left(\mathcal{L}_{1}, C_{2}\right)\right\}$ is indeed minimal in a sense that we do not wish to make precise here but that depends on the order of $k$ and $i$ in the input. To give a sense of this order, observe that the output of creative_telescoping $(f(t, i, k),[i:: \operatorname{shift}, k::$ shift $], t::$ diff $)$ also consists of two pairs $(\mathcal{L}, C)$, but the second one involves solely $S_{i}$.

\section{Convolution with line segments}

We examine the convolution of line segments for power inverse kernels, with varying radius or scale $^{5}$. First we express the convolution functions, with varying radius or scale, in terms of an integral function indexed by two integers:

$$
\boldsymbol{I}_{i, k}(a, b, c, \lambda, \delta)=\int_{-1}^{1} \frac{(\lambda+\delta t)^{k}}{\left(a t^{2}-2 b t+c\right)^{i}} d t
$$

We then provide recurrence formulae on this integral so as to have all the convolution functions for line segments with (even) power inverse kernels. The recurrence relationships we exhibit can be adapted to work for all powers. Furthermore, though we do not give details, these recurence also allow to deal with the convolution of line segments with the family of compact support kernels, by taking $i$ to be a negative integer. We choose to restrict here to even powers as they provide easier formulae to evaluate (odd power inverse kernels bring out elliptic functions in the convolution of arcs of circles and planar polygons). This does not affect too much the variety of shapes we can obtain.

\subsection{Integrals for convolution}

Two points $A, B \in \mathbb{R}^{3}$ define the line segment $[A B]$. A regular parametrization for this line segment is given by $\Gamma:[-1,1] \rightarrow \mathbb{R}^{3}$ with $\Gamma(t)=\frac{A+B}{2}+\frac{B-A}{2} t$. Therefore for a point $P \in \mathbb{R}^{3}$ we have

$$
4|P \Gamma(t)|^{2}=|A B|^{2} t^{2}-2 \overrightarrow{A B} \cdot \overrightarrow{C P} t+|C P|^{2} \text { where } C=\frac{A+B}{2}
$$

is the mid point of the line segment $[A B]$. Hence $\left|\Gamma^{\prime}(t)\right|=\frac{|A B|}{2}$.

The simple convolution of this line segment with the power inverse kernel $\boldsymbol{p}^{2 i}$ is thus given by:

$$
\mathcal{C}_{[A B]}^{2 i}(P)=\frac{|A B|}{2} \int_{-1}^{1} \frac{1}{|P \Gamma(t)|^{2 i}} \mathrm{~d} t=\frac{|A B|}{2} \boldsymbol{I}_{i, 0}\left(\frac{1}{4}|A B|^{2}, \frac{1}{4} \overrightarrow{A B} \cdot \overrightarrow{C P}, \frac{1}{4}|C P|^{2}, \lambda, \delta\right) .
$$

\footnotetext{
${ }^{5}$ Results for convolution of weighted line segments with power inverse and Cauchy kernels can be found in [19].
} 
If we choose the radius function $\rho:[a, b] \rightarrow \mathbb{R}$ to be linear in the arclength we can find $\lambda, \delta \in \mathbb{R}$ such that $\rho(t)=\lambda+\delta t$. Convolution with varying radius is then given by:

$$
\mathcal{H}_{[A B], \rho}^{2 i}(P)=\int_{-1}^{1} \frac{(\lambda+\delta t)^{2 i}}{|P \Gamma(t)|^{2 i}} \frac{|A B|}{2} \mathrm{~d} t=\frac{|A B|}{2} \boldsymbol{I}_{i, 2 i}\left(\frac{1}{4}|A B|^{2}, \frac{1}{4} \overrightarrow{A B} \cdot \overrightarrow{C P}, \frac{1}{4}|C P|^{2}, \lambda, \delta\right) .
$$

If we now take the scale function to be $\Lambda(t)=\lambda+\delta t$, then

$$
\mathcal{S}_{[A B], \Lambda}^{2 i}(P)=\int_{-1}^{1} \frac{(\lambda+\delta t)^{2 i}}{|P \Gamma(t)|^{2 i}} \frac{|A B|}{2(\lambda+\delta t)} \mathrm{d} t=\frac{|A B|}{2} \boldsymbol{I}_{i, 2 i-1}\left(\frac{1}{4}|A B|^{2}, \frac{1}{4} \overrightarrow{A B} \cdot \overrightarrow{C P}, \frac{1}{4}|C P|^{2}, \lambda, \delta\right) .
$$

\subsection{Closed forms through recurrence formulae}

First of all, given that

$$
\boldsymbol{I}_{1,0}(a, b, c, \lambda, \delta)=\frac{1}{\sqrt{a c-b^{2}}}\left[\arctan \left(\frac{a t-b}{\sqrt{a c-b^{2}}}\right)\right]_{-1}^{1}
$$

we can determine $\boldsymbol{I}_{i, 0}(a, b, c, \lambda, \delta)$ for all $i \geq 1$ thanks to the recurrence relationship

$$
2 i\left(a c-b^{2}\right) \boldsymbol{I}_{i+1,0}+(1-2 i) a \boldsymbol{I}_{i, 0}=\left[\frac{a t-b}{\left(a t^{2}-2 b t+c\right)^{i}}\right]_{-1}^{1} .
$$

One then observes that:

$$
2 a(1-i) \boldsymbol{I}_{i, 1}=2(3-i)(a \lambda+b \delta) \boldsymbol{I}_{i, 0}-\left[\frac{\delta}{\left(a t^{2}-2 b t+c\right)^{i-1}}\right]_{-1}^{1}
$$

This recurrence is actually obtained by specializing the following recurrence to $k=-1$

$a(k-2 i+3) \boldsymbol{I}_{i, k+2}+2(i-2-k)(a \lambda+b \delta) \boldsymbol{I}_{i, k+1}+(k+1)\left(a \lambda^{2}+c \delta^{2}+2 b \lambda \delta\right) \boldsymbol{I}_{i, k}=\left[\frac{-\delta(\lambda+\delta t)^{k+1}}{\left(a t^{2}-2 b t+c\right)^{i-1}}\right]_{-1}^{1}$

One can thus determine $\boldsymbol{I}_{i, k}$ for all $i \geq 1$ and $k \geq 0$ and therefore $\boldsymbol{I}_{i, 2 i}$ and $\boldsymbol{I}_{i, 2 i-1}$ that are needed for convolution with varying radius or scale.

Alternatively, to determine the convolution with varying radius, we can consider the recurrence

$$
\begin{array}{r}
2 i(i+1) a\left(a c-b^{2}\right) \boldsymbol{I}_{i+2,2 i+4}-i\left(\lambda a(1+2 i)(2 b \delta+a \lambda)+\left((4 i+5) c a-2(i+2) b^{2}\right) \delta^{2}\right) \boldsymbol{I}_{i+1,2 i+2} \\
+\delta^{2}(i+1)(1+2 i)\left(a \lambda^{2}+c \delta^{2}+2 b \lambda \delta\right) \boldsymbol{I}_{i, 2 i}=\left[\frac{(\lambda+\delta t)^{2 i+1}}{\left(a t^{2}-2 b t+c\right)^{i+1}} C\right]_{-1}^{1}
\end{array}
$$

where

$$
\begin{aligned}
C= & \left(\left(a c+2 b^{2} i\right) t^{2}-b c(3 i+2) t+c^{2}(i+1)\right) \delta^{3} \\
& +\left(2 a b(1+2 i) t^{2}-\left(2(i+2) b^{2}+3 a i c\right) t+b c(i+2)\right) \lambda \delta^{2} \\
& +\left(a^{2}(1+2 i) t^{2}-a b(i+2) t-a c(i-1)\right) \lambda^{2} \delta+a i(a t-b) \lambda^{3}
\end{aligned}
$$

A similar recurrence can be obtained for $\boldsymbol{I}_{i, 2 i-1}$. As the previous ones, it is obtained by Creative Telescoping (see Section 3). 


\section{Convolution with arcs of circle}

We examine the convolution of an arc of circle for power inverse kernels. Though arcs of circles appear in the literature about convolution surfaces [21,38], there is no general formulae for these. In this section we choose a parametrization for arcs of circle that allows us to write the convolution functions (with varying radius or scale) in terms of an integral indexed by two integers:

$$
\boldsymbol{F}_{i, k}(a, b, c, \lambda, \delta)=\int_{-T}^{T} \frac{(\lambda+\delta t)^{k}\left(t^{2}+1\right)^{i-1}}{\left(a t^{2}-2 b t+c\right)^{i}} d t
$$

We then show how to determine closed form formulae for these integrals thanks to some recurrences. We restrict our attention to convolution of arcs of circle with even power inverse kernels. With odd power inverse kernels, the closed form formulae for the convolution function involve elliptic functions and can be rather impractical to evaluate.

The closed form formulae for the convolution of arcs of circle with the family of compact support kernels are challenging to obtain. The software MixedCT ${ }^{6}$ by L. Dumont (in Maple) does meet this challenge. The result would be too cumbersome to be presented here and it is not clear at this stage how to use it efficiently.

\section{$5.1 \quad$ Rational parametrization}

When it comes to integration, rational functions are the dependable class [8]. The main ingredient in obtaining closed-form convolution functions for arcs of circle is to introduce an appropriate rational parametrization.

We assume that the points $O, A$ and $B$ are not aligned and such that $|O A|=|O B|=r$. They define a plane in space and two arcs of circle, one of angle $\alpha$ the other of angle $\pi+\alpha$ for some $0<\alpha<\pi$. We have

$$
\alpha=\arccos \left(\frac{\overrightarrow{O A} \cdot \overrightarrow{O B}}{r^{2}}\right) \quad \text { with } \quad 0<\alpha<\pi
$$

and accordingly to which angle is dealt with we set

$$
T=\tan \left(\frac{\alpha}{4}\right) \quad \text { or } \quad T=\tan \left(\frac{\pi+\alpha}{4}\right) .
$$

Momentarily we consider the coordinate system $(x, y, z)$ where the origin is the center of the circle, the $x$-axis is the bisector of the chosen angle defined by $O, A$ and $B$ and the $(x, y)$ plane is the plane of the circle. See Figure 4.

A parametrization of the arc of circle is then given by

$$
\begin{aligned}
\Gamma:[-T, T] & \rightarrow \mathbb{R}^{3} \\
t & \mapsto\left(r \frac{1-t^{2}}{t^{2}+1}, r \frac{2 t}{t^{2}+1}, 0\right) .
\end{aligned}
$$

\footnotetext{
${ }^{6}$ http://mixedct.gforge.inria.fr
} 


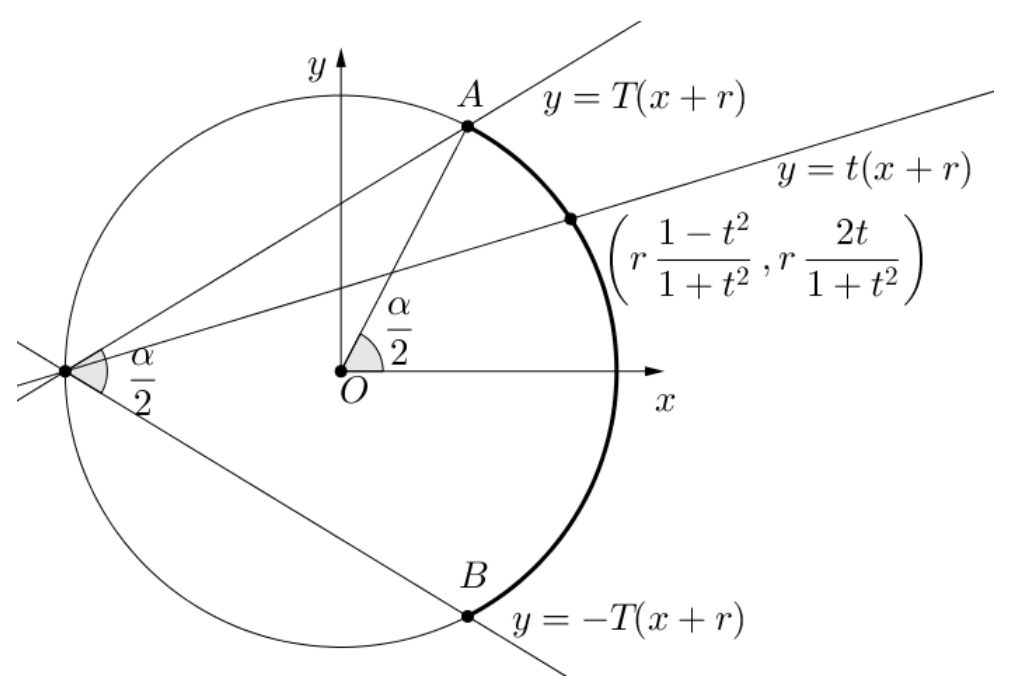

Figure 4: Rational parametrization of an arc of circle.

This is obtained by determining the intersection of the circle with the lines of slope $t$ through the point diametrically opposite to the middle of the arc. Consider a point $P=(x, y, z)$ in space. We have

$|P \Gamma(t)|^{2}=\frac{\alpha t^{2}-2 \beta t+\gamma}{t^{2}+1} \quad$ where $\quad \alpha=(x+r)^{2}+y^{2}+z^{2}, \quad \beta=2 r y, \quad \gamma=(x-r)^{2}+y^{2}+z^{2}$.

Note that

$$
\begin{aligned}
\gamma+\alpha & =2\left(|O P|^{2}+r^{2}\right) \\
\alpha T^{2}+2 \beta T+\gamma & =\left(T^{2}+1\right)|A P|^{2} \\
\gamma T^{2}-2 \beta T+\gamma & =\left(T^{2}+1\right)|B P|^{2}
\end{aligned}
$$

so that $(\alpha, \beta, \gamma)$ is actually the solution of a linear system that depends on $T$ and the squares of the distances of $P$ to $O, A$ and $B$. There is a unique solution provided that $A, O$ and $B$ are not aligned, i.e. $T\left(T^{2}-1\right) \neq 0$. This solution is:

$$
\alpha=\frac{\left(|P A|^{2}+|P B|^{2}\right)\left(T^{2}+1\right)-4\left(|P O|^{2}+r^{2}\right)}{T^{2}-1},
$$

and

$$
\beta=\frac{\left(|P A|^{2}-|P B|^{2}\right)\left(T^{2}+1\right)}{T}, \quad \gamma=2\left(|P O|^{2}+r^{2}\right)-\alpha
$$

\subsection{Integrals for convolution}

Using the above parametrization of an arc of circle $\widehat{A^{O B}}$ the associated convolution function with the power inverse kernel $\boldsymbol{p}^{2 i}$ is

$$
\mathcal{C}_{\overparen{A^{O B}}}^{2 i}(P)=\int_{-T}^{T} \frac{1}{|P \Gamma(t)|^{2 i}} \frac{2 r}{1+t^{2}} \mathrm{~d} t=2 r \int_{-T}^{T} \frac{\left(1+t^{2}\right)^{i-1}}{\left(\alpha t^{2}-2 \beta t+\gamma\right)^{i}} \mathrm{~d} t=2 r \boldsymbol{F}_{i, 0}(\alpha, \beta, \gamma, \lambda, \delta)
$$


as the infinitesimal arc-length is $\left|\Gamma^{\prime}(t)\right|=\frac{2 r}{1+t^{2}}$.

We choose a radius or scale function $\rho, \Lambda:[-T, T] \rightarrow \mathbb{R}$ that is linear in the parameter $t$ used above. A more intrinsic choice would be to have a radius or scale function linear in the arc length. Since the arc-length is $2 r \arctan (t) \sim 2 r t+O\left(t^{3}\right)$, linearity in $t$ is a reasonable approximation for arcs defined by an angle less than $\pi$. This is illustrated in Figure 5.

Convolution with varying radius according to $\rho: t \mapsto \lambda+\delta t$ is then given by

$$
\mathcal{H}_{\overparen{A^{O} B}, \rho}^{2 i}(P)=\int_{-T}^{T} \frac{(\lambda+\delta t)^{2 i}}{|P \Gamma(t)|^{2 i}} \frac{2 r}{1+t^{2}} \mathrm{~d} t=2 r \boldsymbol{F}_{i, 2 i}(\alpha, \beta, \gamma, \lambda, \delta) .
$$

Convolution with scale function $\Lambda: t \mapsto \lambda+\delta t$ is given by

$$
\mathcal{S}_{\overparen{A^{O} B}, \Lambda}^{2 i}(P)=\int_{-T}^{T} \frac{(\lambda+\delta t)^{2 i}}{|P \Gamma(t)|^{2 i}} \frac{2 r}{1+t^{2}} \frac{\mathrm{d} t}{1+\delta t}=2 r \boldsymbol{F}_{i, 2 i-1}^{-T, T}(\alpha, \beta, \gamma, \lambda, \delta) .
$$

\subsection{Closed forms through recurrence formulae}

Given that

$$
\boldsymbol{F}_{1,0}=\left[\frac{1}{\sqrt{c a-b^{2}}} \arctan \left(\frac{a t-b}{\sqrt{c a-b^{2}}}\right)\right]_{-T}^{T}
$$

we can recover the expression for $\boldsymbol{F}_{i, 0}$, for all $i \in \mathbb{N}$, thanks to the recurrence relationship

$2(i+1)\left(a c-b^{2}\right) \boldsymbol{F}_{i+2,0}-(1+2 i)(a+c) \boldsymbol{F}_{i+1,0}+2 i \boldsymbol{F}_{i, 0}=\left[\frac{\left(1+t^{2}\right)^{i}\left(b\left(t^{2}-1\right)+(a-c) t\right)}{\left(a t^{2}-2 b t+c\right)^{i+1}}\right]_{-T}^{T}$

On the other hand, the integrals $\boldsymbol{F}_{i, k}$ satisfy the following recurrence:

$a(k+3) \boldsymbol{F}_{i, k+4}-A_{3} \boldsymbol{F}_{i, k+3}+A_{2} \boldsymbol{F}_{i, k+2}+A_{1} \boldsymbol{F}_{i, k+1}+A_{0}(k+1) \boldsymbol{F}_{i, k}=\left[\frac{\delta^{3}\left(1+t^{2}\right)^{i}(\lambda+\delta t)^{k+1}}{\left(a t^{2}-2 b t+c\right)^{i-1}}\right]_{-T}^{T}$

where

$$
\begin{aligned}
& A_{3}=-2(2 k+5) a \lambda-2 b(i+2+k) \delta, \\
& A_{2}=6(k+2) a \lambda^{2}+2 b(5+3 k+2 i) \delta \lambda+((3+k-2 i) a+(k+2 i+1) c) \delta^{2}, \\
& A_{1}=-2 a(3+2 k) \lambda^{3}-2 b(4+3 k+i) \delta \lambda^{2}+2((i-2-k) a-(i+1+k) c) \delta^{2} \lambda+2 b(i-2-k) \delta^{3}, \\
& A_{0}=\left(\delta^{2}+\lambda^{2}\right)\left(\lambda^{2} a+c \delta^{2}+2 b \lambda \delta\right) .
\end{aligned}
$$

By specializing the above equation to $k=-1$ one can obtain $\boldsymbol{F}_{i, 3}$ from $\boldsymbol{F}_{i, 2}, \boldsymbol{F}_{i, 1}$ and $\boldsymbol{F}_{i, 0}$. These latter are thus sufficient to determine $\boldsymbol{F}_{i, k}$ for all $k \geq 4$.

To determine $\boldsymbol{F}_{i, 1}$ we observe that, for $i \neq 0$,

$$
a \boldsymbol{F}_{i+1,1}-\boldsymbol{F}_{i, 1}=(b \delta+a \lambda) \boldsymbol{F}_{i+1,0}-\lambda \boldsymbol{F}_{i, 0}-\frac{\delta}{2 i}\left[\frac{\left(1+t^{2}\right)^{\frac{i}{2}}}{\left(a t^{2}-2 b t+c\right)^{\frac{i}{2}}}\right]_{-T}^{T}
$$


and

$$
\boldsymbol{F}_{1,1}=\left[\frac{(b \delta+a \lambda)}{a \sqrt{a c-b^{2}}} \arctan \left(\frac{a t-b}{\sqrt{a c-b^{2}}}\right)+\frac{\delta}{2 a} \ln \left(a t^{2}-2 b t+c\right)\right]_{-T}^{T} .
$$

To determine $\boldsymbol{F}_{i, 2}$ we can use the linear recurrence that provides $\boldsymbol{F}_{i, k+2}$ in terms of $\boldsymbol{F}_{i, k+1}$, $\boldsymbol{F}_{i, k}, \boldsymbol{F}_{i+1, k}$, and $\boldsymbol{F}_{i+2, k}$. Specialized to $k=0$ this recurrence simplifies to:

$$
A_{02} \boldsymbol{F}_{i, 2}+A_{01} \boldsymbol{F}_{i, 1}+A_{00} \boldsymbol{F}_{i, 0}+A_{10} \boldsymbol{F}_{i+1,0}+A_{20} \boldsymbol{F}_{i+2,0}=\left[\delta \frac{(\lambda+\delta t)\left(1+t^{2}\right)^{i}}{\left(a t^{2}-2 b t+c\right)^{i+1}} C\right]_{-T}^{T}
$$

where

$$
\begin{aligned}
A_{02}= & a\left((a-c) \delta \lambda+b\left(\delta^{2}-\lambda^{2}\right)\right) \\
A_{01}= & -2(i b \delta+\lambda a)\left(-\lambda^{2} b+(a-c) \delta \lambda+b \delta^{2}\right) \\
A_{00}= & -\left(\delta^{2}+\lambda^{2}\right)\left(b((2 i-1) a+2 c i) \delta^{2}+\left((2 i-1) a^{2}+a c+2 b^{2} i\right) \lambda \delta+\lambda^{2} a b\right), \\
A_{10}= & (1+2 i) a^{2}(a+c) \delta \lambda^{3}+b\left(3 a^{2}(1+2 i)+a c(3+4 i)+2 i b^{2}\right) \delta^{2} \lambda^{2} \\
& +\left((4 i+1) c a^{2}+a\left(c^{2}+2 b^{2}(i+1)\right)+2 b^{2}(3 i+1) c\right) \delta^{3} \lambda+b\left((4 i+1) c a+(1+2 i) c^{2}-2 i b^{2}\right) \delta^{4}, \\
A_{20}= & -2 \delta(i+1)(\lambda a+b \delta)\left(a c-b^{2}\right)\left(a \lambda^{2}+c \delta^{2}+2 b \lambda \delta\right)
\end{aligned}
$$

and

$$
\begin{aligned}
C= & a^{2}\left(b-b t^{2}-(a-c) t\right) \lambda^{2}+\left(a^{2} b t^{2}-b^{2}(3 a+c) t+b\left(c^{2}+2 b^{2}\right)\right) \delta^{2} \\
& +\left(a^{2}(a-c) t^{2}-2 b\left(b^{2}+2 a^{2}-a c\right) t+b^{2}(3 a+c)\right) \delta \lambda
\end{aligned}
$$

\section{Application and outlook}

In this section we illustrate the benefit that arcs of circle provide as basic elements of skeletons. Arcs of circle have desirable approximation power for space curves with high curvature or torsion. The formulae we presented thus allow to use them to generate convolution functions and surfaces: each arc of circle, or line segment, in the skeleton generates a convolution function through the formulae presented in Section 5, or 4, and the convolution function generated by the skeleton is the sum of all of these. In the examples presented here we used the kernel $\boldsymbol{p}^{6}$ and Hornus formulation (defined in Section 2.5.1) to generate the convolution functions. The convolution surfaces presented are the level set at value 0.1 of the obtained convolution functions.

Figure 5 shows the convolution with a varying radius of a line segment and four arcs of circles. The skeleton curves all have the same extremities but different radius. The angle supporting the arc of circle thus varies. Only when this angle is close to $2 \pi$ does one detect visually that the thickness does not vary linearly with the arc length. For each skeleton piece $\Gamma([a, b])$, the radius function $\rho:[a, b] \rightarrow \mathbb{R}$ is given by an affine function (in the parameter of the parametrization of $\Gamma$ ) with $\rho(a)=1$ and $\rho(b)=4$. The segments are given by an arc-length parametrization of length 25 . The arcs are given by the rational parametrization introduced in Section 5.1 (Figure 4), the radius was chosen to give a chord length of 25 for each example in Figure 5.

We can combine several arcs of circles and line segments to model $\mathcal{G}^{1}$ curves to serve as skeleton. This is illustrated for two closed curves in Figure 6. 

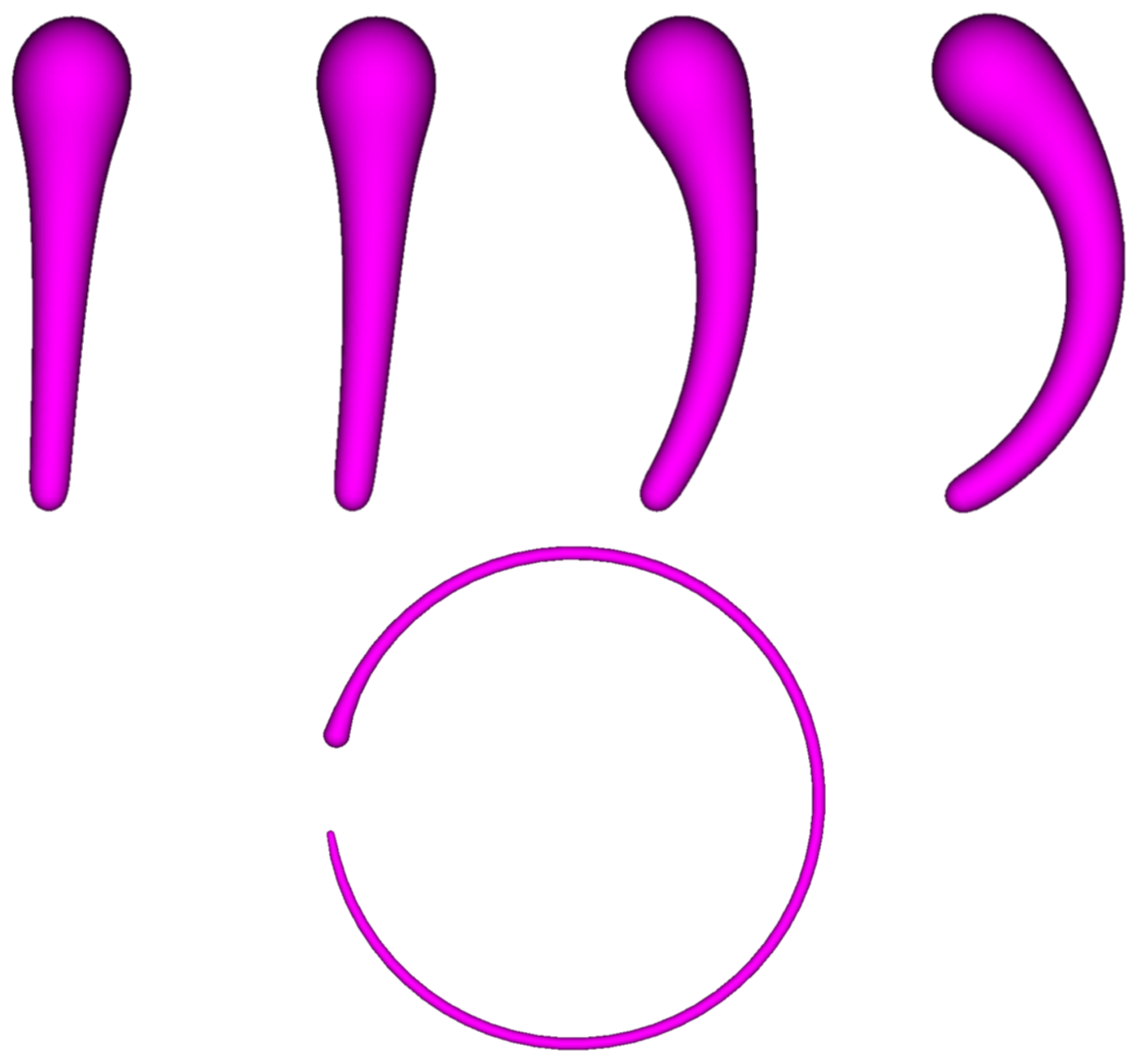

Figure 5: Convolution with varying radius for a line segment (top left) and arcs of circles supported respectively by an angle $\frac{\pi}{24}, \frac{\pi}{3}, \frac{2 \pi}{3}$ and $\frac{19 \pi}{10}$ (bottom, with a different scale).

The widely used approach for more elaborate skeleton curves is to use an approximation by line segments $[9,17,22,33,37]$. An issue with this versatile approach is that either the resulting convolution surface presents some visible turns at the joints of line segments, or the number of segments must be increased significantly in order to get a visually smooth surface. Convolution for arcs of circles were also examined in [21, 38]. [21] dealt with planar skeletons. [38] was geared towards skeleton curves with nonzero torsion: arcs of circles, and line segments, were deformed into helices that have powerful modeling properties for natural shapes, in particular for the animation of hair [3]. The warping technique used in [38] allows to decrease substantially the number of skeleton basic elements to be used to obtain a natural looking shape. This provides a substantial gain on the computational cost as meshing the surface requires the repeated evaluation of the convolution function. Yet the surfaces obtained by warping in [38] exhibit artifacts and singularities so that this technique requires a fine tuning of the warping parameters.

The alternative approach we want to bring forth in this paper is to use a $\mathcal{G}^{1}$-approximation of 

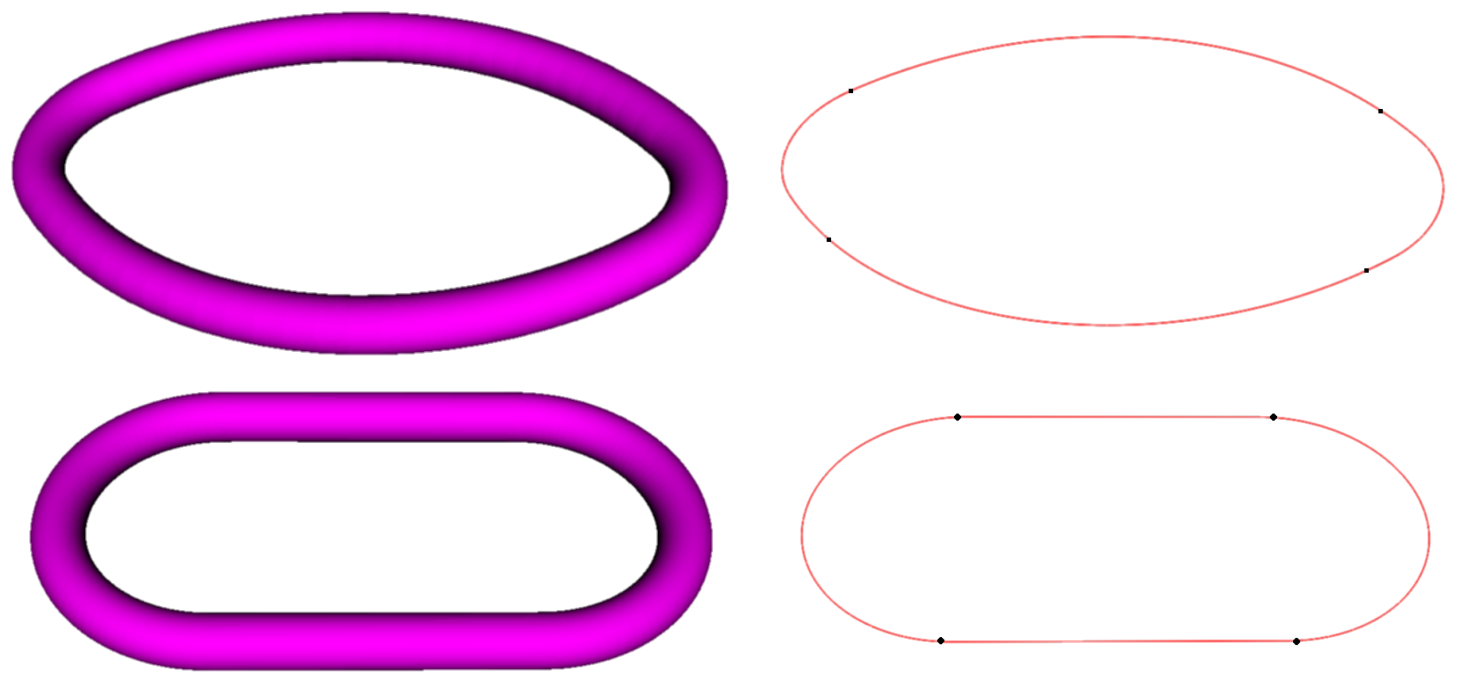

Figure 6: Convolution surface modeling a smooth chain ring. Top row: modeling with arcs of circle only; bottom: arcs of circle and line segments. Left image: the surface; right: the skeleton composed of only 4 arcs of circle; in black the joint points.

the skeleton curve. Arcs of circles have the great advantage to allow the construction of $\mathcal{G}^{1}$-curves that can approximate any curve $[28,34]$. One can thus achieve both mathematically smooth and visually appealing shapes with skeleton curves consisting of few basic elements. This improves visual quality and decreases the computational cost. Figures 7,8 and 9 compare the convolution surfaces with skeleton curves generated with arcs of circle and line segments. The visual quality of the surface is obtained with much fewer arcs of circles rather than line segments.

Conclusion: In this paper we have focused on convolution surfaces along skeleton consisting of a single curve, either open or closed. We propose to use approximation by line segments and arcs of circles to approximate this curve so as to obtain quality convolution surfaces with lower computational cost. To this effect we provided explicit formulae for the convolution functions for both line segments and arcs of circles, with varying radii or scale functions. These formulae have great generality that draws from the use of recurrence formulae that were obtained with a new technique, creative telescoping.

The great advantage of convolution is to provide a practical mathematical definition of a smooth surface around complex skeletons made of intersecting curves and surfaces. Contrary to offset, sweep or canal surfaces, convolution surfaces naturally blend smoothly multiple primitive shapes. There are nonetheless challenges in their use. First the visualization mostly relies on refined marching cube algorithms. An alternative approach would be based on the prior construction of a scaffold around the skeleton as introduced in [30], with an alternative approach in [15]. A second challenge is the control of the topology and geometry. This problem was tackled in $[26,36]$. We expect to provide an alternative more intrinsic formulation, with mathematical guarantees. 


$$
\begin{aligned}
& \text { ss } \\
& w
\end{aligned}
$$


$\infty \otimes$ 


\section{References}

[1] Alexe, A., Barthe, L., Cani, M.-P., And Gaildrat, V. Shape Modeling by Sketching using Convolution Surfaces. In 13th Pacific Graphics Short papers (Macau, China, Oct. 2005).

[2] Bernhardt, A., Pinuit, A., Cani, M.-P., and Barthe, L. Matisse : Painting 2D regions for Modeling Free-Form Shapes. In Eurographics Workshop on Sketch-Based Interfaces and Modeling (SBIM) (Annecy, France, June 2008), pp. 57-64.

[3] Bertails, F., Audoly, B., Cani, M.-P., Querleux, B., Leroy, F., And Lévêque, J.-L. Super-helices for predicting the dynamics of natural hair. ACM Transactions on Graphics 25, 3 (2006), 1180.

[4] Blinn, J. F. A Generalization of Algebraic Surface Drawing. ACM Transactions on Graphics 1, 3 (1982), 235-256.

[5] Bloomenthal, J., And Shommake, K. Convolution surfaces. ACM SIGGRAPH Computer Graphics 25, 4 (jul 1991), 251-256.

[6] Bostan, A., Chen, S., Chyzak, F., Li, Z., and Xin, G. Hermite reduction and creative telescoping for hyperexponential functions. In ISSAC 2013-Proceedings of the 38th International Symposium on Symbolic and Algebraic Computation. ACM, New York, 2013, pp. 77-84.

[7] Bostan, A., Dumont, L., And Salvy, B. Efficient Algorithms for Mixed Creative Telscoping. In Proceedings of the ACM on International Symposium on Symbolic and Algebraic Computation (New York, NY, USA, 2016), ISSAC '16, ACM, pp. 127-134.

[8] Bronstein, M. Symbolic Integration I, vol. 1 of Algorithms and Computation in Mathematics. Springer Berlin Heidelberg, Berlin, Heidelberg, 1997.

[9] Cani, M. P., And Hornus, S. Subdivision-curve primitives: A new solution for interactive implicit modeling. In Proceedings - International Conference on Shape Modeling and Applications, SMI 2001 (2001), pp. 82-88.

[10] Cani, M.-P., Igarashi, T., And Wyvill, G. Interactive Shape Design. Synthesis Lectures on Computer Graphics and Animation. Morgan \& Claypool Publishers, 2008.

[11] Chen, S., And Kauers, M. Trading order for degree in creative telescoping. Journal of Symbolic Computation 47, 8 (2012), 968 - 995.

[12] Chen, S., And Kauers, M. Some open problems related to creative telescoping. Journal of Systems Science and Complexity 30, 1 (Feb 2017), 154-172.

[13] Chyzak, F. An extension of Zeilberger's fast algorithm to general holonomic functions. Discrete Mathematics 217, 1 (2000), 115-134.

[14] Chyzak, F., And Salvy, B. Non-commutative Elimination in Ore Algebras Proves Multivariate Identities. Journal of Symbolic Computation 26, 2 (1998), 187-227.

[15] Fuentes SuÁrez, A. J., And Hubert, E. Scaffolding skeletons using spherical Voronoi diagrams. Electronic Notes in Discrete Mathematics 62 (2017), 45-50. 
[16] He, Q., Tong, M., And Liu, Y. Face Modeling and Wrinkle Simulation Using Convolution Surface. Springer Berlin Heidelberg, Berlin, Heidelberg, 2006, pp. 244-251.

[17] Hornus, S., Angelidis, A., And Cani, M.-P. Implicit modeling using subdivision curves. The Visual Computer 19, 2 (2003), 94-104.

[18] Hubert, E. Convolution surfaces based on polygons for infinite and compact support kernels. Graphical Models 74, 1 (2012), 1-13.

[19] Hubert, E., And Cani, M.-P. Convolution surfaces based on polygonal curve skeletons. Journal of Symbolic Computation 47, 6 (2012), 680-699.

[20] Jin, X., AND TAI, C.-L. Analytical methods for polynomial weighted convolution surfaces with various kernels. Computers and Graphics 26 (2002), 437-447.

[21] Jin, X., AND TAI, C. L. Convolution surfaces for arcs and quadratic curves with a varying kernel. Visual Computer 18, 8 (2002), 530-546.

[22] Jin, X., Tai, C.-L., Feng, J., and Peng, Q. Convolution Surfaces for Line Skeletons with Polynomial Weight Distributions. ACM Journal of Graphics Tools 6, 3 (2001), 17-28.

[23] Jin, X., TAI, C.-L., AND Zhang, H. Implicit modeling from polygon soup using convolution. The Visual Computer 25, 3 (2009), 279-288.

[24] Koutschan, C. A fast approach to creative telescoping. In Mathematics in Computer Science (2010).

[25] Koutschan, C. Creative Telescoping for Holonomic Functions. Springer Vienna, Vienna, 2013, pp. 171-194.

[26] Ma, G., And Crawford, R. H. Topological Consistency in Skeletal Modeling With Convolution Surfaces. In Volume 3: 28th Computers and Information in Engineering Conference, Parts $A$ and $B$ (2008), ASME, pp. 307-315.

[27] Milnor, J. W. Morse Theory. 153.

[28] Nutbourne, A. W., And Martin, R. R. Differential geometry applied to curve and surface design. John Wiley \& Sons, 1988.

[29] Ore, O. Theory of Non-Commutative Polynomials. The Annals of Mathematics 34, 3 (jul 1933), 480.

[30] Panotopoulou, A., Welker, K., Ross, E., Hubert, E., and Morin, G. Scaffolding a Skeleton.

[31] Piessens, R., de Doncker-Kapenga, E., Überhuber, C. W., And Kahaner, D. K. Quadpack, vol. 1 of Springer Series in Computational Mathematics. Springer Berlin Heidelberg, Berlin, Heidelberg, 1983.

[32] Sherstyuk, A. Interactive shape design with convolution surfaces. In Shape Modeling International '99 (1999), pp. 56-65. 
[33] Sherstyuk, A. Kernel functions in convolution surfaces: A comparative analysis. Visual Computer 15, 4 (1999), 171-182.

[34] Song, X., Aigner, M., Chen, F., And Jüttler, B. Circular spline fitting using an evolution process. Journal of Computational and Applied Mathematics 231, 1 (2009), 423-433.

[35] Wyvill, G., McPheeters, C., And Wyvill, B. Data structure for soft objects. The Visual Computer 2, 4 (1986), 227-234.

[36] Zanni, C. Skeleton-based Implicit Modeling \& Applications. Phd, Université de Grenoble, 2013.

[37] Zanni, C., Bernhardt, A., Quiblier, M., and Cani, M.-P. SCALe-invariant integral surfaces. Computer Graphics Forum 32, 8 (2013), 219-232.

[38] Zanni, C., Hubert, E., And Cani, M.-P. Warp-based helical implicit primitives. Computers and Graphics 35, 3 (2011), 517-523.

[39] Zhu, X., Jin, X., Liu, S., And Zhao, H. Analytical solutions for sketch-based convolution surface modeling on the gpu. The Visual Computer 28, 11 (Nov 2012), 1115-1125.

[40] Zhu, X., Jin, X., And You, L. High-quality tree structures modelling using local convolution surface approximation. Visual Computer 31, 1 (2015), 69-82. cited By 1.

[41] Zhu, X., Song, L., You, L., Zhu, M., Wang, X., And Jin, X. Brush2model: Convolution surface-based brushes for 3d modelling in head-mounted display-based virtual environments. Computer Animation and Virtual Worlds 28, 3-4 (2017), e1764-n/a. e1764 cav.1764. 